\title{
Protective effect of recombinant Trichinella 53-kDa protein in sepsis and the effect on macrophages
}

\author{
CHUWEI YANG* ${ }^{*}$ HUI LIU* ${ }^{*}$ XIANGDONG LI, SHAOGUANG SUI and YUFEI LIU \\ Emergency Center, The Second Hospital of Dalian Medical University, Dalian, Liaoning 116023, P.R. China
}

Received December 24, 2015; Accepted May 17, 2016

DOI: $10.3892 /$ etm.2016.3354

\begin{abstract}
The survival rate of the recombinant Trichinella 53-kDa protein infected by the cecal ligation and puncture (CLP) model of sepsis in rats and the expression difference of macrophages were analyzed. Eighteen clean SD rats were selected for the present study. The rats were divided into the sham operation $(n=5)$, control $(n=5)$ and experimental $(n=8)$ groups. The rats in the sham operation group underwent cecum division and suture with routine therapy for cure. The rats in the control and experimental groups were placed in the CLP model of sepsis in rats. The experimental group was administered recombinant Trichinella 53-kDa protein in advance, and the control group was administered the same dose of placebo. The survival rate of the rats within 6 and $12 \mathrm{~h}$, the macrophage expression ratio, and the differences of the expression levels Th1-type cytokines IFN- $\gamma$ and tumor necrosis factor (TNF)- $\alpha$, and the Th2 type cytokines interleukin-4 (IL-4) and IL-13 were analyzed. The survival rate of rats in the experimental group was higher than that of the control group with a statistically significant difference $(\mathrm{P}<0.05)$. The expression ratio of the macrophages received from the different handling methods of the rats in the experimental group was higher than that of the control group. The difference was statistically significant $(\mathrm{P}<0.05)$. The expression levels of the Th1-type cytokines IFN- $\gamma$ and TNF- $\alpha$ of the rats in the experimental group was higher than that of the control group, while the expression level of the Th2-type cytokines IL-4 and IL-13 was higher than that of the control group. The difference was statistically significant $(\mathrm{P}<0.05)$. In conclusion, recombinant Trichinella 53-kDa protein can increase the survival rate following infection with CLP sepsis, which may be associated with the improvement of the macrophages and the adjustment of the expression of Th2 cytokines.
\end{abstract}

Correspondence to: Dr Yufei Liu, Emergency Center, The Second Hospital of Dalian Medical University, 467 Zhongshan Road, Shahekou, Dalian, Liaoning 116023, P.R. China

E-mail: 1_yf2013@163.com

*Contributed equally

Key words: recombinant Trichinella 53-kDa protein, sepsis, cecal ligature and puncture, macrophage, anti-inflammatory

\section{Introduction}

In clinical settings, sepsis always occurs secondary to severe infection, trauma, burn, and major surgery. Severe sepsis and septic shock can lead to multiple organ dysfunction syndromes and death. The annual death frequency caused by sepsis can reach 100,000 worldwide, and the cost of treatment can reach tens of billions of dollars (1). The abnormal immune mechanism in sepsis has become a hot research topic and macrophages that play an important role in antigen presentation in the process of organism immunity, constitute a communication bridge. According to the condition of induced differentiation, macrophages can be classified to M1 type or M2 macrophage, both of which have different activation pathways, producing a series of cytokines and inducing organism immune response to change toward different directions after activation (2). Previous findings have shown that Th2 immune response is enhanced in patients with trichinellosis, even manifesting as immunosuppression. This type of change can remit the occurrence of immune disease (3). Due to high risk and potentially lethal trichinization, the application of the recombinant Trichinella protein may bring new research directions for use in immune diseases (4).

\section{Materials and methods}

Experimental animals. Eighteen clean Sprague-Dawley (SD) rats (Shanghai Shenggong Technology Co., Ltd., Shanghai, China) from the same race and brood, aged 6-8 weeks with a weight of $190 \pm 10 \mathrm{~g}$, were selected and randomly divided into the sham operation $(n=5)$, control $(n=5)$ and experimental $(n=8)$ groups. Differences pertaining to comparison among age, weight and health state were not statistically significant $(\mathrm{P}>0.05)$. The rats in the sham operation group underwent cecum division and suture with routine therapy for cure. The rats in the control and experimental groups underwent cecal ligation and puncture (CLP). The experimental group was given recombinant Trichinella 53-kDa protein in advance, and the control group was given the same dose of placebo.

Preparation of the CLP rat model. Preoperative abrosia was carried out for $12 \mathrm{~h}$, with isoflurane inhalation anesthesia to induce and maintain the anesthesia state of the rats. The rats were placed in supine position, the skin of hypogastrium was disinfected with iodine, and the rats underwent direct shear 
operation to cut the skin, muscular layer and peritoneum gradually below the middle of linea alba approximately $0.3 \mathrm{~cm}$. Using a tweezer the muscle layer was lifted to explore the abdominal cavity, and the end of appendix was removed and placed outside of the abdominal wall, then ligatured $1.6 \mathrm{~cm}$ from the cecum end with the suture line. Two holes were punctured vertically to the cecum, which was then drained. The cecum was returned to the abdominal cavity and the abdomen was sutured. An immediate neck and back subcutaneous injection of $50 \mathrm{ml} / \mathrm{kg}$ compound sodium chloride was carried out and the rats were placed in the cage. The rats were given water and food after $4 \mathrm{~h}$. The animals were kept indoors at a temperature of $20 \pm 1{ }^{\circ} \mathrm{C}$, humidity $60 \pm 10 \%$ and light from 6:00 a.m. to 6:00 p.m. (5).

Approval for the animal studies was received from the ethics committee of the Second Hospital of Dalian Medical University.

Preparation and depuration of decombinant Trichinella 53-kDa protein. The prepared pET-28a(+)-TsP53-E. coli BL21 glycerine bacteria were defrosted, inoculated and merged in $3 \mathrm{ml} \mathrm{LB}$ liquid medium for culture. Kanamycin solution (3 $\mu 1$ ) was then added, single distributed bacteria were selected for cloning, and the culture was agitated for $12 \mathrm{~h}$. When the culture medium was turbid, the liquid was obtained and the culture continued. Subsequently, $3 \mathrm{ml} 1 \mathrm{M}$ IPTG inducer was added, followed by lysating it on ice using ultrasound pyrolysis apparatus (Nanjing Xianou Instrument Manufacturing Co., Ltd., Nanjing, China), the supernatant was discarded and the sediment was kept after three cycles of centrifugation at 3,000 x g for $20 \mathrm{~min}$. Then, $30 \mathrm{ml} 8 \mathrm{M}$ urea solution was added to the sediment and was resuspended, which was placed on a shaker to clear the liquid. The supernatant was obtained by filtering the $0.45 \mu \mathrm{m}$ microporous membrane (Xi'an DingChang Co., Ltd., Xi'an, China), as the chromatographic sample of Ni-NTA-HIS metal chelate. The dialyzed protein sample was added to the ToxinEraser ${ }^{\mathrm{TM}}$ endotoxin to remove the resin chelating for $1 \mathrm{~h}$, prior to being eluted. The process was repeated to condense the rTsP53 protein sample (6).

Preparation of rat anti-rTsP53 protein polyclonal antibody. A quantity of $200 \mu \mathrm{g}$ purified rTsP53 protein sample was emulsified in Freund's complete adjuvant, which was then injected into the SD rats subcutaneously, at 3 and 5 weeks. The peripheral blood of the animals was kept and the rTsP53 protein antibody was detected using ELISA (7).

Detection of bone marrow-derived macrophages (BMDMs) $M 1$ and M2. The rats were sacrificed by cervical dislocation, and an annular incision was performed under the abdominal skin of rats, with the lower limb skin by blunt separation. The knee joint was cut, and the connection of the hip and spine was examined by careful separation to expose the femur and hip. The partial, residual muscle and tendon tissue were removed. The two ends of the bone were cut, one of which was injected with $2.5 \mathrm{ml}$ Dulbecco's modified Eagle's medium (DMEM) without any sodium pyruvate using a $2.5 \mathrm{ml}$ syringe needle. The marrow tissue was placed in a $15 \mathrm{ml}$ centrifuge tube with medium liquid, until the marrow cavity became white. The supernatant of $30 \%$ L929 cell culture medium and the
Table I. Comparison of the survival rates in three groups of rats $[$ case $(\%)]$.

\begin{tabular}{lccc}
\hline Groups & Cases & $6 \mathrm{~h}(\%)$ & $12 \mathrm{~h}(\%)$ \\
\hline Sham operation & 5 & 5 & 5 \\
Control & 5 & $2(40.0)$ & $1(20.0)$ \\
Experimental & 8 & $6(75.0)$ & $5(62.5)$ \\
$\chi^{2}$ & & 3.925 & 4.152 \\
P-value & & $<0.001$ & $<0.001$ \\
\hline
\end{tabular}

cells were added. The cells were cultivated at $7^{\circ} \mathrm{C}$, in a $5 \%$ $\mathrm{CO}_{2}$ environment for $96 \mathrm{~h}$, changing the liquid and removing the components that did not adhere. Macrophage BMDMs of the bone marrow were obtained (8). Then, induced differentiation of the macrophages were carried out as per the instructions.

The survival rate difference of the rats was analyzed at 6 and $12 \mathrm{~h}$, and proportion of the macrophages expressed among the groups. The expression level of Th1-type cytokines IFN- $\gamma$ and tumor necrosis factor (TNF)- $\alpha$, as well as Th2-type cytokines interleukin-4 (IL-4) and IL-13 were compared.

Statistical analyis. Data were analyzed using SPSS 19.0 statistical software (SPSS Inc. Chicago, IL, USA). Data were presented as mean \pm standard deviation. Comparison between groups were carried out using the t-test. Countable data were expresses as cases or percentage, and comparison of groups were carried out using the Chi-square $\left(\chi^{2}\right)$ test. $\mathrm{P}<0.05$ was statistically significant.

\section{Results}

Comparison of the survival rates in three groups of rats. The survival rate of rats in the experimental group was higher than that of the control group at 6 and $12 \mathrm{~h}$, and the difference was statistically significant $(\mathrm{P}<0.05)$ (Table I).

Comparison of the expression ratio of macrophages. After $72 \mathrm{~h}$ of the blank medium, we obtained PE-CCR7(-), FITC-CD206(-) macrophages (A group). Through the activation of $20 \mathrm{ng} / \mathrm{ml}$ IFN- $\gamma$ for $72 \mathrm{~h}$, we obtained PE-CCR7(+) macrophages (B group). Through the activation of $10 \mathrm{ng} / \mathrm{ml} \mathrm{IL-3/IL-14} \mathrm{for}$ 72 h, we obtained FITC-CD206(+) macrophages (C group); following culture with rTsP53 protein for $72 \mathrm{~h}$, we obtained FITC-CD206(+) macrophages (D group); and following culture of $20 \mu \mathrm{g} / \mathrm{ml} \mathrm{IFN}-\gamma$ and $5 \mu \mathrm{g} / \mathrm{ml}$ rTsP53 protein for $72 \mathrm{~h}$, we obtained PE-CCR7(+) macrophages (E group). The expression ratio of the macrophage in the experimental group was higher than that of the control group, and the difference was statistically significant $(\mathrm{P}<0.05)$ (Table II).

Comparison of the expression level of Th1- and Th2-type cytokines. The expression level of the Th1-type cytokines IFN- $\gamma$ and TNF- $\alpha$ of the experimental group was higher than that of the control group, while the expression level of the Th2-type cytokines IL-4 and IL-13 was higher in the control group. The difference was statistically significant $(\mathrm{P}<0.05)$ (Table III). 
Table II. Comparison of the expression ratio of different macrophages (\%).

\begin{tabular}{|c|c|c|c|c|c|}
\hline Groups & A & B & $\mathrm{C}$ & $\mathrm{D}$ & $\mathrm{E}$ \\
\hline Control & $81.7 \pm 16.4$ & $19.8 \pm 5.2$ & $69.4 \pm 15.5$ & $2.3 \pm 0.2$ & $10.9 \pm 2.4$ \\
\hline Experimental & $92.6 \pm 10.3$ & $32.5 \pm 6.4$ & $82.7 \pm 14.2$ & $5.2 \pm 0.7$ & $16.8 \pm 3.3$ \\
\hline t-test & 3.526 & 3.784 & 4.152 & 4.829 & 4.628 \\
\hline P-value & $<0.001$ & $<0.001$ & $<0.001$ & $<0.001$ & $<0.001$ \\
\hline
\end{tabular}

Table III. Comparison of expression level of Th1- and Th2-type cytokines.

\begin{tabular}{lccrr}
\hline Groups & IFN- $\gamma(\mathrm{ng} / \mathrm{l})$ & TNF- $\alpha(\mathrm{ng} / \mathrm{l})$ & $\mathrm{IL}-4(\mathrm{pg} / \mathrm{ml})$ & $\mathrm{IL}-13(\mathrm{ng} / \mathrm{l})$ \\
\hline Control & $725.6 \pm 51.2$ & $352.8 \pm 25.9$ & $125.3 \pm 15.8$ & $24.6 \pm 5.9$ \\
Experimental & $429.3 \pm 46.3$ & $125.3 \pm 36.4$ & $365.9 \pm 24.3$ & $64.2 \pm 3.4$ \\
t-test & 4.526 & 5.268 & 5.024 & 5.928 \\
P-value & $<0.001$ & $<0.001$ & $<0.001$ & $<0.001$ \\
\hline
\end{tabular}

\section{Discussion}

Clinical studies $(9,10)$ suggesting that an abnormal immune mechanism leading to excessive activation of the organism inflammation/anti-inflammatory reaction process can cause tissue damage or immune paralysis has become an important field of investigation in sepsis research. A new treatment concept of immunomodulation has emerged as a result. Macrophages serve as antigens presenting in the body's immune process, and a series of cytokines that macrophages produce further regulate the body's immune reaction process. Therefore, the study of macrophages and immune response may identify the deeper mechanisms of the occurrence and development of sepsis, leading to new treatments and concepts $(11,12)$.

Function of abnormal immunologic mechanism on sepsis. Anti-inflammatory media have been used to study animals with sepsis, for example, classic TNF- $\alpha$, with the result showing that the anti-inflammatory medium treatment by TNF- $\alpha$ is able to improve the prognosis of partial sepsis in animals (13). At the same time, previous findings showed (14) that besides the classic inflammatory medium, IL-4, IL-10 and IL-13 improved significantly patients with SIRS. Due to the findings of early inflammation/anti-inflammatory medium research, it was found that intervention by single regulation of inflammation/antiinflammatory cytokine did not correct perfectly the prognosis of sepsis. Since a complicated intervention network of each other existed in inflammation/anti-inflammatory cytokines, similar to the waterfall release of inflammatory cytokines in SIRS, intervention at any point of the inflammatory cytokine pathway may lead to unpredictable and unmanageable alteration of the entire pathway. This type of complex neuroendocrine network change in body determines the treatment of sepsis and should not simply utilize the regulation of inflammation/antiinflammatory cytokine $(15,16)$.

Function of macrophage active state on sepsis. In sepsis, macrophage can phagocytose pathogenic microorganisms directly, which breaks pathogenic microorganisms through enzyme and bio-activator that by itself produced changes and promotes the process of immune response in body through the activator and its function of antigen presenting (17). Thus, macrophages are important immune cells that constitute 'a connecting link between the preceding and the following' (18).

A significant feature of macrophages is its obvious heterogeneity, which can form different directions of differentiation according to the micro-environmental state of its tissue. In terms of the conditions of induced differentiation, the original macrophage, also known as M0 macrophage, is formed by the induced differentiation of the early macrophage colony-stimulating factor, which can be divided into classically activated M1 macrophage, and alternatively activated M2 macrophage (19). Each has a different pathway of activation, and a series of cytokines from activation induce an immune response to change towards different directions (20). M1 macrophage, its production of Th1 immune response and other substances after its activation can damage tissue. However, if the M1 macrophage and related Th1 immune response are not regulated, it may lead to the amplification of body inflammatory reaction with SIRS, severe sepsis, and MODS (19). M2 macrophage can be classified as a,b and c according to its function and mechanism, of which, M2a macrophage is activated by IL-4 and IL-13 mainly with the function of promoting wound healing through the generation of extracellular matrix. Thus, IL-4 and IL-13 are the important characteristic cytokines of Th2 immune response (21). As mentioned earlier, in various inflammatory diseases of the body, M1/M2 macrophage can reflect a balanced state of inflammation/anti-inflammatory reaction in the body. M1 macrophage and inflammation reaction can remove the pathogenic microorganism by producing various bioactive substances. The M2 macrophage and antiinflammatory reaction can promote tissue repair and control the inflammatory response, avoiding to some extent the effect of excessive damage (22). The M2 macrophages and antiinflammatory response promote tissue repair and control the 
inflammatory response to a certain extent to avoid the effect of excessive damage (23).

Effect of recombinant Trichinella 53-kDa protein on macrophages. Previous findings have shown (23) that, the enhancement of Th2 immune response or even immunosuppression in patients and animals with sepsis can alleviate the occurrence of certain autoimmune diseases. In a study using chronic trichinization Dark Agouti (DA) rats, it was found that experimental cerebrospinal meningitis was alleviated, and Th2 immune response was activated in the model (19). The secretory protein of Trichinella can influence immunosuppression in vitro, and rTsP53 protein is a type of secretory protein in Trichinella that improves the prognosis of sepsis by activating M2 macrophages in the body (21).

The results of the present study show that the survival rate of rats in the experimental group was higher than that of the control group at 6 and $12 \mathrm{~h}$, and the difference was statistically significant. The expression ratio of the macrophages received from the different handling methods of the rats in the experimental group was higher than that of the control group, and the difference was statistically significant. The expression level of the Th1-type cytokines IFN- $\gamma$ and TNF- $\alpha$ of rats in the experimental group was higher than that of the control group. By contrast, the expression level of the Th2-type cytokines IL-4 and IL-13 was higher than that of the control group, and the difference was statistically significant. Therefore, recombinant Trichinella 53-kDa protein can increase the survival rate after the infection by CLP sepsis in rats, which may be associated with the improvement of the macrophages and the adjustment of the expression of the Th2 cytokines.

\section{References}

1. Maeda JL, Raetzman SO and Friedman BS: What hospital inpatient services contributed the most to the 2001-2006 growth in the cost per case? Health Serv Res 47: 1814-1835, 2012.

2. Lagu T, Rothberg MB, Shieh MS, Pekow PS, Steingrub JS and Lindenauer PK: Hospitalizations, costs, and outcomes of severe sepsis in the United States 2003 to 2007. Crit Care Med 40: 754-761, 2012.

3. Matsuda A, Jacob A, Wu R, Aziz M, Yang WL, Matsutani T, Suzuki H, Furukawa K, Uchida E and Wang P: Novel therapeutic targets for sepsis: regulation of exaggerated inflammatory responses. J Nippon Med Sch 79: 4-18, 2012.

4. Song R, Kim J, Yu D, Park C and Park J: Kinetics of IL-6 and TNF- $\alpha$ changes in a canine model of sepsis induced by endotoxin. Vet Immunol Immunopathol 146: 143-149, 2012.

5. Bhatia M1, He M, Zhang H and Moochhala S: Sepsis as a model of SIRS. Front Biosci (Landmark Ed) 14: 4703-4711, 2009.
6. Wu HP, Wu CL, Chen CK, Chung K, Tseng JC, Liu YC and Chuang DY: The interleukin-4 expression in patients with severe sepsis. J Crit Care 23: 519-524, 2008.

7. Urbonas V, Eidukaite A and Tamulienè I: Increased interleukin-10 levels correlate with bacteremia and sepsis in febrile neutropenia pediatric oncology patients. Cytokine 57: 313-315, 2012.

8. Gustot T: Multiple organ failure in sepsis: prognosis and role of systemic inflammatory response. Curr Opin Crit Care 17: 153-159, 2011.

9. Mills CD: M1 and M2 macrophages: oracles of health and disease. Crit Rev Immunol 32: 463-488, 2012.

10. Martinez FO, Sica A, Mantovani A and Locati M: Macrophage activation and polarization. Front Biosci 13: 453-461, 2008.

11. Pan XQ: The mechanism of the anticancer function of M1 macrophages and their use in the clinic. Chin J Cancer 31: 557-563, 2012.

12. Qin H, Holdbrooks AT, Liu Y, Reynolds SL, Yanagisawa LL and Benveniste EN: SOCS3 deficiency promotes M1 macrophage polarization and inflammation. J Immunol 189: 3439-3448, 2012.

13. Benoit M, Desnues B and Mege JL: Macrophage polarization in bacterial infections. J Immunol 181: 3733-3739, 2008

14. Abdu SB: Schistosoma mansoni induce granulomatous inflammation and lesion to the enteric nervous system in mouse colon. J Egypt Soc Parasitol 39: 183-190, 2009.

15. Babu S, Kumaraswami V and Nutman TB: Alternatively activated and immunoregulatory monocytes in human filarial infections. J Infect Dis 199: 1827-1837, 2009.

16. Bai X, Yu JL, Wang F, Zhao Y, Liu MY and Wang GM: Alternatively activated macrophages in helminth infections. Zhongguo Ji Sheng Chong Xue Yu Ji Sheng Chong Bing Za Zhi 29: 219-223, 2011 (In Chinese).

17. da Silva RF, Lappalainen J, Lee-Rueckert M and Kovanen PT: Conversion of human M-CSF macrophages into foam cells reduces their proinflammatory responses to classical M1-polarizing activation. Atherosclerosis 248: 170-178, 2016.

18. Cui J, Wang ZQ and Xu BL: The epidemiology of human trichinellosis in China during 2004-2009. Acta Trop 118: 1-5, 2011.

19. Gruden-Movsesijan A, Ilic N, Mostarica-Stojkovic M, Stosic-Grujicic S, Milic M and Sofronic-Milosavljevic L: Mechanisms of modulation of experimental autoimmune encephalomyelitis by chronic Trichinella spiralis infection in Dark Agouti rats. Parasite Immunol 32: 450-459, 2010.

20. Knopp S, Steinmann P, Keiser J and Utzinger J: Nematode infections: soil-transmitted helminths and trichinella. Infect Dis Clin North Am 26: 341-358, 2012.

21. Du L, Wei H, Li L, Shan H, Yu Y, Wang Y and Zhang G: Regulation of recombinant Trichinella spiralis $53-\mathrm{kDa}$ protein (rTsP53) on alternatively activated macrophages via STAT6 but not IL-4R $\alpha$ in vitro. Cell Immunol 288: 1-7, 2014.

22. Park HK, Cho MK, Choi SH, Kim YS and Yu HS: Trichinella spiralis: infection reduces airway allergic inflammation in mice. Exp Parasitol 127: 539-544, 2011

23. Bruschi $\mathrm{F}$ and Chiumiento L: Immunomodulation in trichinellosis: does Trichinella really escape the host immune system? Endocr Metab Immune Disord Drug Targets 12: 4-15, 2012. 